Artículo

\title{
Elaboración de objetos de aprendizaje abiertos para ciencias agrícolas bajo la metodología PADDIEM
}

\author{
José Manuel Meraz Escobar ${ }^{1}$ \\ José Luis García Cué ${ }^{1 \S}$ \\ Yolanda M. Fernández Ordóñez ${ }^{1}$ \\ Mercedes A. Jiménez Velázquez ${ }^{1}$ \\ Reyna Carolina Medina Ramírez ${ }^{2}$ \\ Dora Ma. Sangerman-Jarquín ${ }^{3}$
}

${ }^{1}$ Colegio de Postgraduados en Ciencias Agrícolas. Carretera México-Texcoco km 36.5, Montecillo, Texcoco, Estado de México. CP. 56230. Tel. 55 58045900. (manuelmeraz21@gmail.com; yfernand@colpos.mx; mjimenez@colpos.mx). ${ }^{2}$ Unidad Iztapalapa-Universidad Autónoma Metropolitana. San Rafael Atlixco 186, Col. Vicentina, Iztapalapa, México. CP. 09340. Tel. 5558044636. (rcarolinamedina@gmail.com). ${ }^{3}$ Campo experimental Valle de México-INIFAP. Carretera los ReyesTexcoco km 13.5. Coatlinchan, Texcoco, Estado de México, México. CP. 56250. Tel. 55 38718700, ext. 85353. (sangerman.dora@inifap.gob.mx).

${ }^{\S}$ Autor para correspondencia: jlgcue@colpos.mx.

\section{Resumen}

La investigación tuvo por objetivo elaborar objetos de aprendizaje abiertos (OAA) para ciencias agrícolas bajo la metodología PADDIEM. La pesquisa se planteó para trabajar en la creación de Recursos Educativos Abiertos (REA) en formato digital a manera de objetos de aprendizaje elaborados bajo modelos instruccionales con los que no se había trabajado en el Colegio de Postgraduados (CP). El OAA se llevó a cabo en el Colegio de Postgraduados, campus Montecillo durante el primer semestre del año 2018. En la metodología, se comenzó con la elaboración de un esquema con la propuesta de un OAA en tres partes: pedagógica, computacional y diseño gráfico. Después, se analizaron diferentes modelos instruccionales para el diseño de REA. A continuación, se hizo el planteamiento de la metodología de la planeación, análisis, diseño, desarrollo, implementación, evaluación y mantenimiento basada en modelos instruccionales (PADDIEM) por sus siglas en inglés y en buenas prácticas de Ingeniería de Software. En esta parte, se destacó tanto su arquitectura como los equipos multidisciplinarios de cada etapa. En los resultados, se mostraron ejemplos de las fases donde participaron el equipo de diseño gráfico para la elaboración de un OAA del tema de modelo entidad-relación de bases de datos. Para concluir, la metodología PADDIEM propuesta sirvió para la elaboración de OAA para ser utilizados en Ciencias Agrícolas.

Palabras clave: educación, equipos multidisciplinarios, REA.

Recibido: junio de 2019

Aceptado: agosto de 2019 


\section{Introducción}

El Colegio de Postgraduados de México (CP) es una institución de educación, investigación, vinculación y servicio fundada en 1959 para la solución de problemas en áreas agrícolas, pesqueras, ganaderas, sociales, forestales, etc. Para lograr lo anterior, desde 1964, se ha apoyado de diferentes herramientas computacionales establecidas en el Centro de Estadística y Cálculo que actualmente forma parte del Programa de Socioeconomía, Estadística e Informática (PSEI).

Los programas de postgrado de estadística y cómputo aplicado han sido de gran apoyo para los análisis de datos de investigaciones agrícolas y para la propuesta de diferentes proyectos de investigación en áreas de inteligencia artificial, bases de datos, sistemas de información geográficos, dataware house, minería de datos, informática educativa, sistemas expertos, sistemas de información, ingeniería de software, etc.

Desde el año 2014, en el área de informática educativa, se ha dado impulso al trabajo con objetos de aprendizaje (OA) para apoyar cursos en diferentes modalidades apoyadas en tecnologías de la información y la comunicación (TIC) como el b-learning y e-learning. Algunos resultados que se destacan son el sistema gestor de objetos de aprendizaje para ciencias agrícolas (SIGEOACA) de Montes et al. (2017), la propuesta de un sistema gestor de objetos de aprendizaje adaptativos a estilos de aprendizaje (SiGOAA-EA) de García-Cué et al. (2017) y los objetos de aprendizaje abiertos fundamentados en ingeniería de software educativo (OAAISE) para cursos de bases de datos de García-Cué et al. (2018).

En los proyectos de OA anteriormente mencionados, se ha trabajado bajo estructuras y sistemas propuestos por los autores, pero no se ha experimentado sobre objetos de aprendizaje abiertos (OAA) bajo modelos instruccionales establecidos -como el ADDIE (siglas de cinco etapas para el desarrollo de recursos educativos digitales: análisis, desarrollo, diseño, implementación y evaluación) o el modelo PADDIE que agrega, al modelo anterior, una etapa de planeación- que están dentro de los estándares para utilizarse en diversos repositorios y bibliotecas nacionales.

También, se propone una nueva metodología de elaboración de OAA propia para el CP que sirva para ciencias agrícolas y que integre modelos instruccionales, buenas prácticas de ingeniería de software y la inclusión de equipos multidisciplinarios -como de educación para el desarrollo de habilidades didácticas para entornos virtuales, diseñadores gráficos, expertos en cómputo, entre otros- como parte fundamental de los OAA. Para cumplir con lo anterior, se planteó una metodología que incluyó las seis etapas del modelo PADDIE más una de mantenimiento (M) dando como resultado PADDIEM.

Para comenzar la investigación, se identificaron las diferentes perspectivas de distintos autores sobre el término objeto de aprendizaje. Wiley (2008); Correa (2017) coincidieron en que los OA forman parte de recursos tecnológicos estructurados como programación orientada a objetos (POO). Cacheiro (2011), con un enfoque a la educación, los tipificó como recursos educativos TIC de aprendizaje.

Portillo (2017) definió a los OA como recursos TIC que deben incluir estrategias didácticas para el aprendizaje significativo como las pre-instruccionales, co-instruccionales y posinstruccionales. Wiley (2008) los definió como cualquier recurso digital que pueden ser reutilizado para apoyar el 
aprendizaje. Sobre las características que debe tener un OA, diversos autores como Moreiro et al. (2012) destacan accesibilidad, granularidad, interoperabilidad, durabilidad y escalabilidad y que además sean relevantes y reutilizables.

Por otro lado, García-Cué et al. (2017) explicaron que los OA pueden estar diseñados según su nivel de globalidad y lo expresaron en tres capas: curso completo, temático y específico. Wiley (2008); Soto (2011); Montes et al. (2017) propusieron otra configuración de OA contemplando dos partes: la computacional y la parte pedagógica. Meraz et al. (2018) agregaron una tercera parte: diseño gráfico como un elemento que posibilita la creación de OA de mejor calidad.

La UNESCO (2012) explicó que los objetos de aprendizaje abiertos (OAA) son catalogados como parte de los recursos educativos abiertos (REA) donde cualquier persona puede tener acceso a ellos sin ningún costo y que además su uso sea público. Para ello, formalizó la declaración de París de REA en el año 2012, donde hizo un llamado a los gobiernos de todo el mundo para que doten de licencias abiertas, como Comunes Creativos (Creative Commons - CC) o licencia pública general reducida (con sus siglas en inglés GNU GPL) a materiales educativos digitales, incluyendo los OAA con fondos financiados por el estado respetando los derechos de las personas que los elaboraron (UNESCO, 2015).

Después, García-Cué et al. (2018); Meraz et al. (2018) prefirieron hablar de procesos y no de partes, ya que éstos son una serie de etapas que se tienen que llevar a cabo para construir los OAA. Además, destacaron tres de ellos.

Proceso pedagógico: para describir de manera lógica y secuencial cada una de las acciones que deben realizarse en la elaboración de un OAA para que sea didáctico y que además influya en el proceso enseñanza-aprendizaje del alumno (García-Aretio, 2013). Lo anterior, se logra por dos caminos. El primero, a través de estructuras de OA propuestas por diferentes autores como Osondón y Castillo (2006); Montes et al. (2017); García-Cué et al. (2017), donde se deben cumplir con al menos introducción, objetivos, teoría, actividades, evaluación y colaboración.

El segundo, apoyados de las etapas de diseños instruccionales para la elaboración de software educativo como el ADDIE (DDEU, 1975 en Meraz et al. 2018), Paddie (Navedtra, 2010), PADDIE+M o una propuesta propia. Además, este proceso incluye al experto en el área del conocimiento en que se desarrollará.

Proceso computacional: para la programación de elementos, el manejo de servidores y redes en internet, la digitalización de cada uno de los elementos que forman parte del OAA (texto, fotografías, documentos, videos, audios, etc.) como sugieren Clares (2011); Meraz et al. (2018). Algunos autores como García-Cue et al. (2018) destacaron la estructura informática basada en diseños instruccionales sustentados en buenas prácticas de ingeniería de software bajo distintos modelos (Cascada, Espiral, XP, SCRUM, etc.) como el ADDIE o PADDIE. También, Moreiro et al. (2012) resaltan en este proceso, el manejo de la parte semántica a manera de metadatos bajo estándares internacionales como: IEEE-LOM, SCORM, CISCO, DCMI, DUBLIN CORE, OAI-PMH, entre otros.

Proceso de diseño gráfico: para la organización, producción y elaboración de cada uno de los materiales que van a formar parte del OAA a manera de plantillas, textos, audios, videos, juegos, etc. (Chan, 2006; García-Cué et al., 2018; Meraz et al., 2018). La función del diseñador es 
encargarse de la parte visual. estética, identidad corporativa y de teorías de percepción para coadyuvar en el proceso enseñanza-aprendizaje apoyado de teorías de ergonomía cognitiva (Gónzalez-Muñoz, 2017) y además centrados en el usuario final, la relación humano-máquina; a través, de interfaces (desde sencillas hasta avanzadas), las estrategias de producto y propuestas para la mercadotecnia del OAA (Pratt y Nunez, 2012).

Además, Clares (2011); García-Cué et al. (2018); Meraz et al. (2018) destacaron la necesidad de integrar grupos de personas expertas en cada proceso o conocedoras de más de un proceso.

Después de analizar todo lo anterior, surgió la siguiente pregunta: ¿cómo se elaboran objetos de aprendizaje abierto para la enseñanza de ciencias agrícolas bajo una metodología PADDIEM? Para contestar esta pregunta se hizo una investigación que tuvo por objetivo elaborar objetos de aprendizaje abiertos (OAA) para Ciencias Agrícolas bajo la metodología PADDIEM. El supuesto planteado fue 'el diseño gráfico y la metodología PADDIEM sirven para la elaboración de objetos de aprendizaje abiertos para ciencias agrícolas'. El trabajo se inscribe en el campo de la investigación educativa incorporando avances de las ciencias y en tecnologías de la información y la comunicación (TIC).

Los resultados de esta investigación servirán como una guía para elaborar OAA para cualquier área del conocimiento en ciencias agrícolas y que además puedan ser utilizados en bibliotecas digitales nacionales. Importante es mencionar que se explicarán cada una de las etapas del PADDIEM, pero en este documento se hace mayor énfasis a la etapa de diseño.

\section{Metodología}

La metodología de los objetos de aprendizaje abiertos está fundamentada en diseño gráfico y se llevó a cabo de la siguiente manera:

- Se elaboró un esquema con la propuesta de un OAA en tres partes: pedagógica, computacional y diseño gráfico. El esquema se realizó a manera de conjuntos con sus correspondientes intersecciones. En cada una de ellas, se resaltaron los conocimientos que deben tener las personas que se integran como equipos multidisciplinarios.

- Se analizó el modelo ADDIE. En esta parte, se explicó este modelo desde su aparición. También, se hizo una breve descripción de sus etapas porque son base de la propuesta metodológica para el CP.

- Se planteó la metodología PADDIEM. En esta parte, se mostró su fundamentación y la propuesta de su arquitectura. También, se explicó cada una de las fases de PADDIEM donde se incluyeron las actividades en cada etapa y el equipo multidisciplinario que interviene.

- Se destacaron tanto el proceso como algunas habilidades del diseñador gráfico para la elaboración de materiales digitales de los OAA dentro de PADDIEM.

- Se mostraron algunos ejemplos de materiales producidos para el OAA basados en los procesos de diseño gráfico. 


\section{Resultados}

\section{Esquema de objetos de aprendizaje abiertos}

La Figura 1, muestra una propuesta de los tres procesos que intervienen en la elaboración de OAA: pedagógicos, computacionales y diseño gráfico.

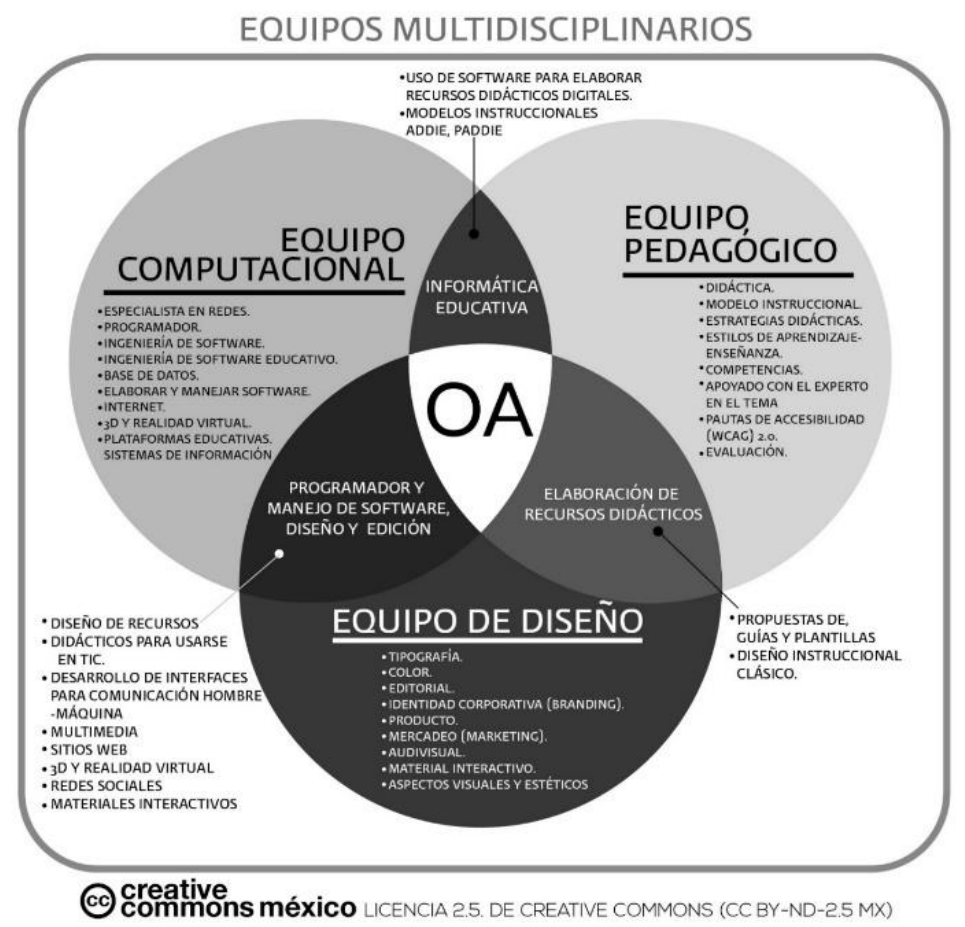

Figura 1. Propuesta de estructura de OAA.

Cada circunferencia representa un proceso, su equipo de expertos correspondiente y el tipo de conocimiento que se recomienda que deban de tener. También hay tres intercepciones entre dos procesos con personas que conocen más de un área de experiencia, al que se le asignó un nombre o actividades que desarrollan. La intercepción de los tres procesos da como resultado un objeto de aprendizaje. La inclusión de la leyenda Creative Commons (CC BY-ND-2.5 MX) es para indicar que es un recurso educativo abierto que se adapta a los lineamientos establecidos en México y que es utilizado por diferentes desarrolladores de OA que ya están disponibles en Internet.

Importante es mencionar que las funciones de los equipos no son rígidas ya que puede haber personas que tengan experiencia de distintas áreas del conocimiento para la elaboración de los OAA. También, puede haber otras que aporten distintos elementos que no están contempladas en el diagrama y que sirven para la elaboración de los OAA. Pratt y Nunez (2012); Leal-Fonseca (2008) coinciden en la necesidad de trabajar un proyecto con equipos multidisciplinarios para la elaboración de materiales didácticos educativos de calidad destacando al diseñador gráfico como un elemento importante. 
Lo anterior, coincide a la propuesta elaborada en este diagrama para el diseño de OAA, solo que en la Figura 1 se proponen los conocimientos que deben tener los especialistas de cada área. Diversos autores, como Clares (2011); Montes et al. (2017), explican que son los docentes los que se encargan de hacer todo para los OA, muchas veces apoyados por software o por plataformas de OA para su creación, sin tomar en cuenta las tres áreas de expertos que se destacan.

\section{Modelo ADDIE}

DDEU, García Cué et al. 2018) explicaron que en 1975 apareció el modelo ADDIE, como una mejora del modelo Instructional System Design (ISD), propuesto cómo sistemas de información de materiales educativos a través de medios computacionales, desarrollado por el Ejército de EE. UU para dar adiestramiento masivo al personal militar que era mandado a la guerra. Branson et al. (2018) explicaron que el Centro de Tecnología Educativa de la Universidad Estatal de Florida (FSU) hizo las modificaciones basándose en propuestas de modelos de Ingeniería de Software y explicó que ADDIE es un acrónimo de distintas fases en el diseño de recursos pedagógicos para medios digitales.

La Figura 2 muestra un diagrama de flujo del modelo ADDIE modificado de los originales de MENC (2014) y CODAES (2015). En dicha figura, aparecen rombos con la palabra AUTORIZA que son etapas de evaluación.

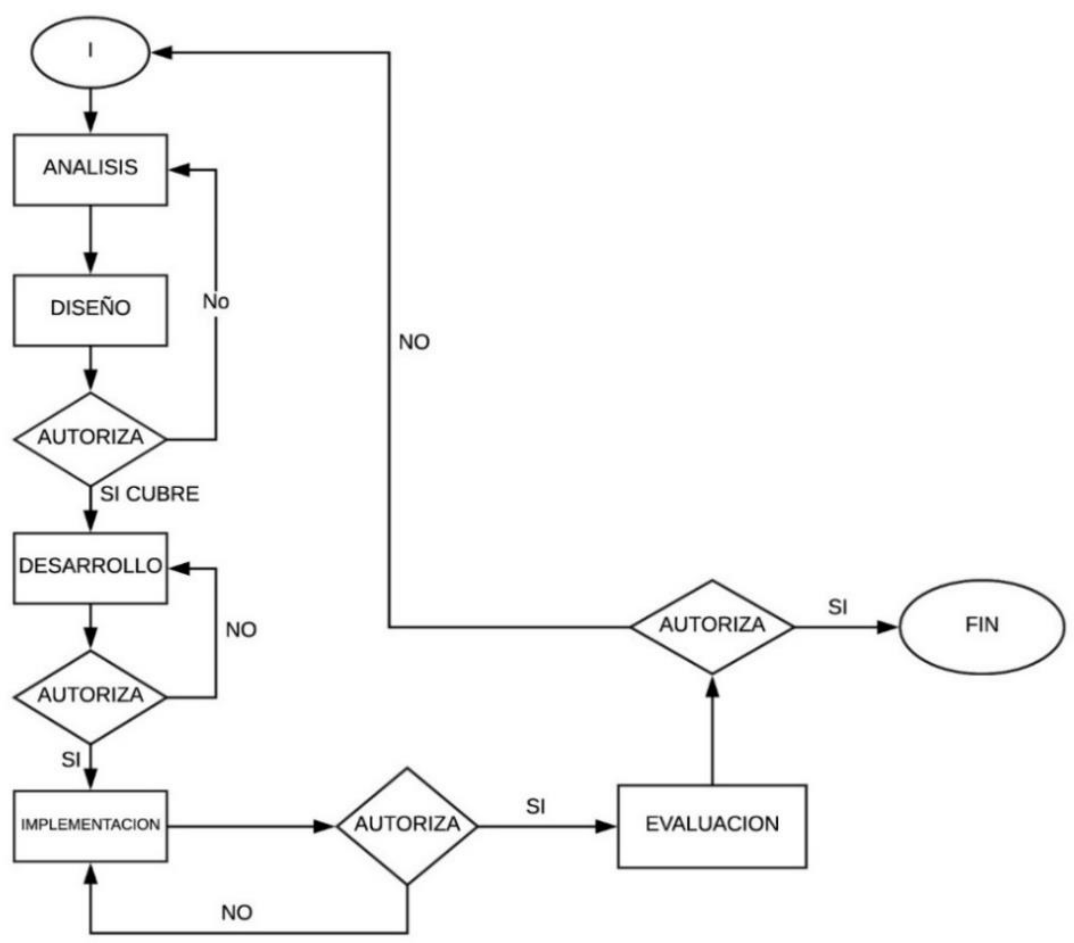

\section{Figura 2. Modelo ADDIE.}

García Cué et al. (2018); Meraz et al. (2018) explicaron cada una las etapas ADDIE para los OA:

Análisis. Se definen los objetivos, así como se recogen los datos necesarios para el diseño de un objeto de aprendizaje. También, se definen características de la audiencia que hará uso del OA. 
Diseño. Se escriben los objetivos, se planea la estructura y la secuenciación del objeto de aprendizaje. También, se crea un plan de gestión del proyecto de OA. Además, se evalúan las etapas de análisis y diseño.

Desarrollo. Se plantea cada uno de los elementos que formarán parte del OA a manera de textos (con teorías, ejemplos, ejercicios, etc.), elementos de reflexión, guiones, juegos, gráficos, audio, video e imágenes. Al término de esta etapa, se procede a una evaluación de todos los materiales antes de implementarlos.

Implementación. Se integra todo lo que lleva el recurso educativo o curso. Se proponen los metadatos basados en estándares internacionales (SCORM o IEEE-LOM). Además, se hacen las pruebas piloto y evaluación del funcionamiento, del contenido y del desarrollo del OA, para poder publicarlo.

Evaluación. Se evalúa la efectividad del recurso educativo para saber si se han cumplido con los objetivos de su proyecto. La evaluación se hace con cuestionarios o con diferentes rúbricas para cada una de las etapas del ADDIE.

En la actualidad, el modelo ADDIE sigue siendo utilizado para la elaboración de Recursos Educativos Abiertos como los OAA (García Cué et al., 2018) y hay diversos manuales en Internet que explican a detalle cada una de sus fases como el de MENC (2014) y el NAVEDTRA (2010).

\section{Planteamiento de PADDIEM, arquitectura y fases}

El planteamiento de PADDIEM es resultado de la integración de las propuestas sobre el modelo ADDIE de DDEU (1975, en García Cué et al. 2018), la fase de planeación (P) del PADDIE (NAVEDTRA, 2010) y de buenas prácticas de Ingeniería de Software sobre la etapa de Mantenimiento (M). La Figura 3 muestra la propuesta de arquitectura. Nótese, que hay un rombo con la letra E que indica que después de cada etapa se hace una evaluación.

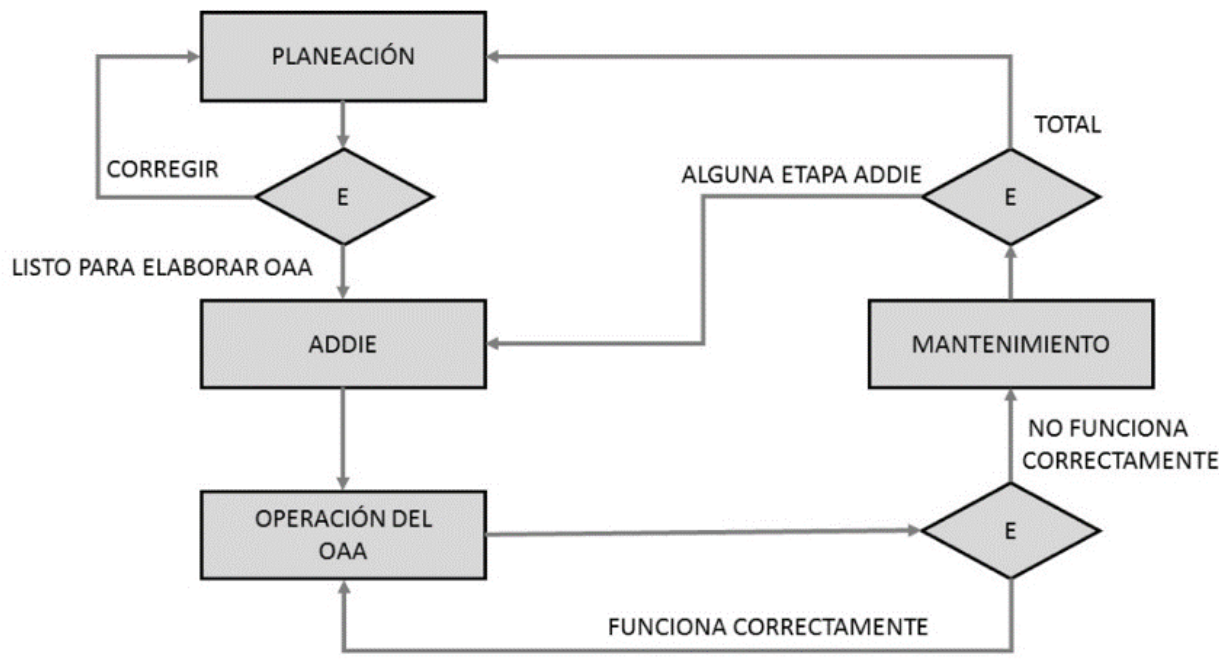

Figura 3. Propuesta de arquitectura del PADDIEM. 
De la figura anterior, se explica lo siguiente:

La fase de planeación (P) identifica las necesidades de recursos, la problemática a solucionar (diagnóstico inicial, viabilidad tanto económica como de recursos humanos, alcance del proyecto, oferta, demanda, usuario final) y la secuencia de acontecimientos en el proceso de desarrollo a manera de cronograma.

Se procede a una evaluación de la planeación (P). Si la evaluación es positiva, se lleva a cabo cada una de las etapas del Modelo ADDIE. Al terminar, se pone en operación el objeto de aprendizaje abierto (OAA).

Se evalúa el funcionamiento de OAA. Si se detectan fallas o se desea corregir los contenidos entra a la parte de mantenimiento (M) donde se toma la decisión de modificarlo todo o se detecta en qué etapa de ADDIE hay que corregirlo.

En la Figura 4, se explica cada una de las etapas de PADDIEM con sus actividades y los equipos multidisciplinarios que se proponen para la elaboración OAA basados en la Figura 1.

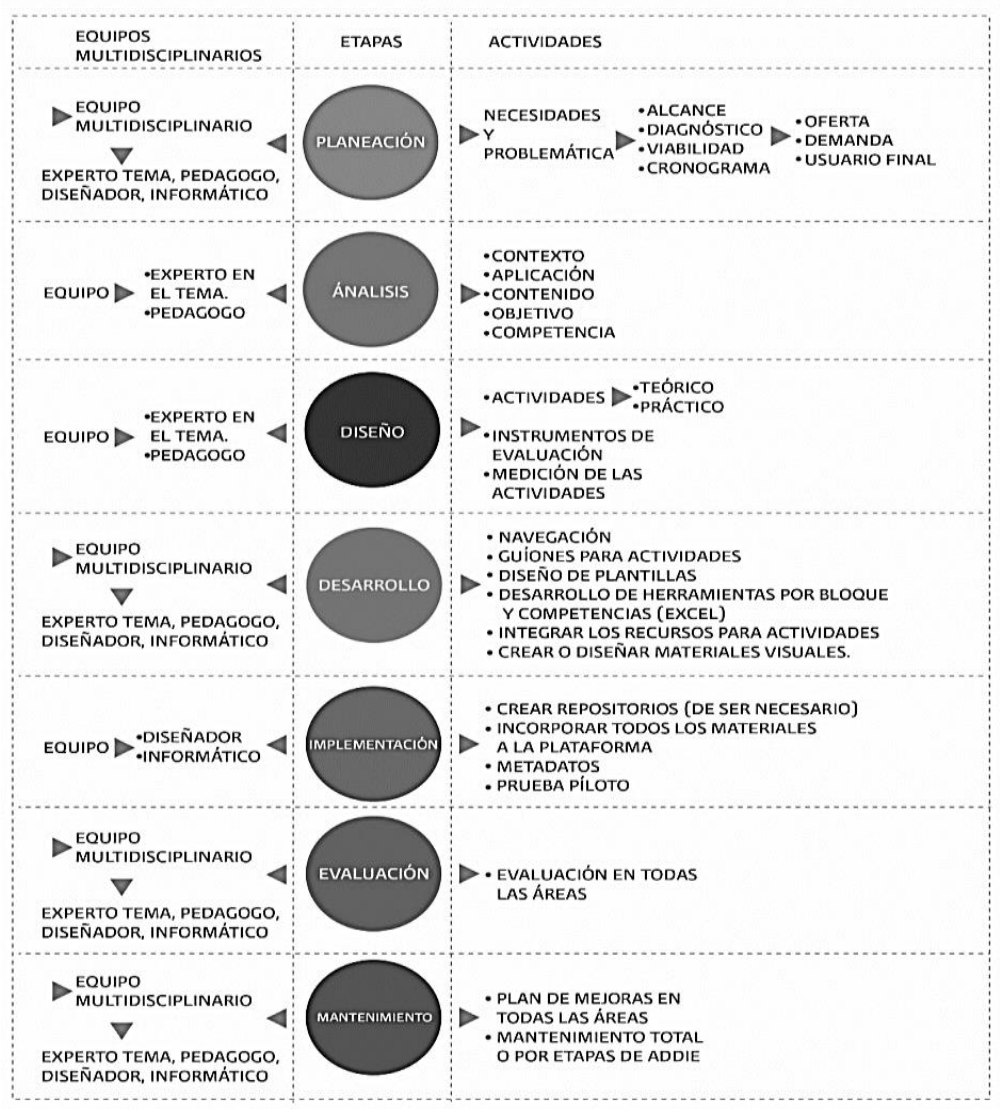

Figura 4. Propuesta de PADDIEM.

El PADDIEM propuesto es similar al de NAVEDTRA (2010), en ambos se explican las actividades de cada fase. Se diferencian en que NAVEDTRA (2010) no considera a los equipos multidisciplinarios, en especial los de diseño gráfico. 
Ordoñez et al. (2015) identificó a su modelo como educator for special group (ESG), que consistía en un ADDIE+M, donde propuso un mantenimiento sugerido en grupos de trabajo y no como se propone en Ingeniería de Software como se hace en el PADDIEM.

Algunos autores mencionan a las etapas de planeación y mantenimiento como algo externo a ADDIE y resaltan que no son esenciales ya que se pueden utilizar o no. En la actualidad, el modelo ADDIE sigue siendo utilizado para la elaboración de OAA (García-Cué et al. 2018), pero diversos autores han propuesto algunas mejoras o adaptaciones de acuerdo con sus propias necesidades de desarrollo de software, muchas de ellas no obligatorias en el proceso.

\section{Proceso y habilidades del diseñador gráfico para la elaboración de OAA en PADDIEM}

Para ejemplificar el modelo PADDIEM, solo se destacará las etapas donde intervienen el grupo de trabajo de los diseñadores gráficos, sus habilidades, así como algunas de sus actividades para el desarrollo un objeto de aprendizaje del tema del modelo entidad-relación de bases de datos agrícolas:

Planeación. Participa activamente en cada una de las decisiones del proyecto, en especial aquellas relacionadas con la estética, contenido, rutas de navegación y los usuarios al que se dirigirá el OAA.

Desarrollo. Propone, junto con lo demás equipos de trabajo, un mapa de navegación de los materiales seleccionados para el OAA, por ejemplo, para el tema de modelo entidad-relación de bases de datos (Figura 5). Después, participa en el desarrollo de cada uno de los materiales, ya sean digitales, interactivos o impresos. Además, tanto los equipos de diseño como los computacionales trabajan en las interfaces para una adecuada comunicación humano-máquina.

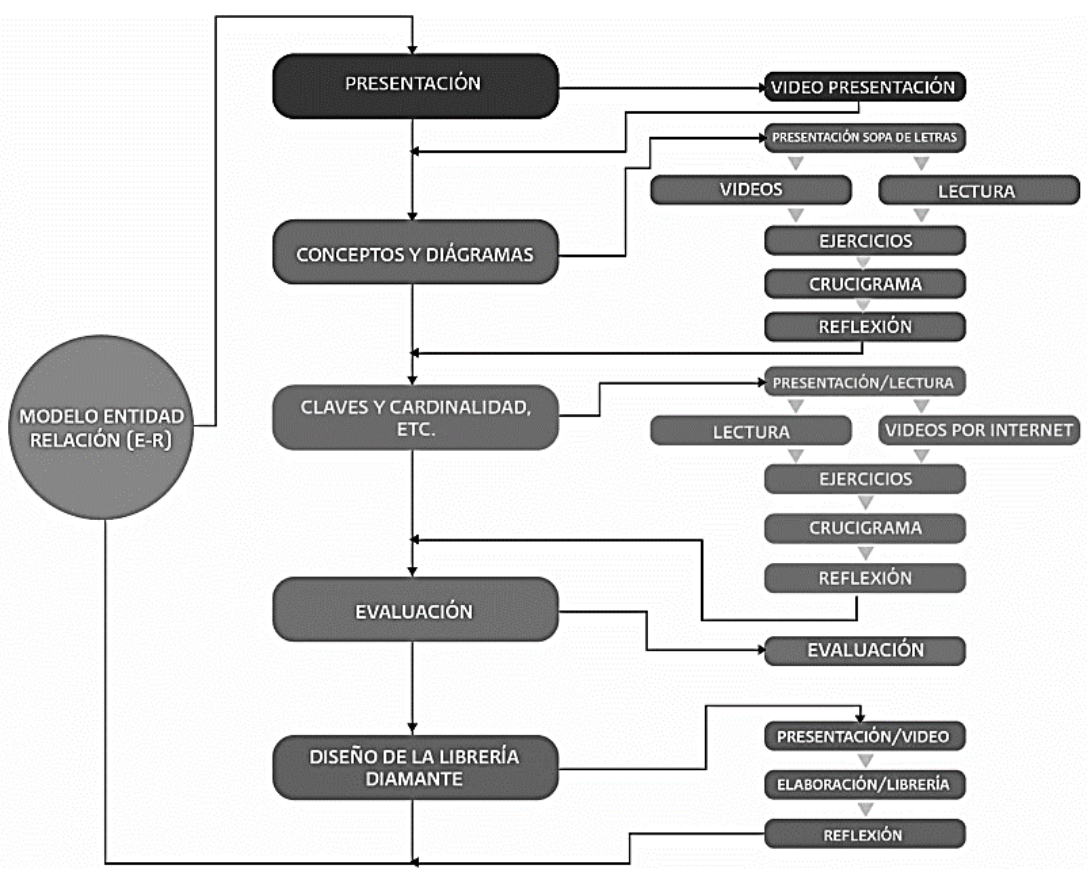

Figura 5. Materiales propuestos para el OAA y mapa de navegación. 
Implementación. Trabaja junto con el experto computacional para adaptar los materiales a los repositorios de OAA.

Evaluación. Participa en la elaboración de instrumentos para verificar si el OAA es adecuado al proyecto.

Mantenimiento. Verifica el buen funcionamiento del OAA. También, corrige errores tipográficos, de estilo, visuales, auditivos, etc. Actualiza los nuevos materiales y colabora con todo el equipo multidisciplinario para que el OAA esté funcionando adecuadamente.

Un ejemplo de la labor del diseñador gráfico en la etapa de desarrollo se muestra en la Figura 6, que contiene los diferentes pasos que se deben seguir para la elaboración de materiales educativos digitales. Se inicia con la elección del tema, recolección de información, organización de la información (todo por medio de carpetas y por formatos de archivos, ejemplos, fotos, videos, audios, archivos de texto, etc.).

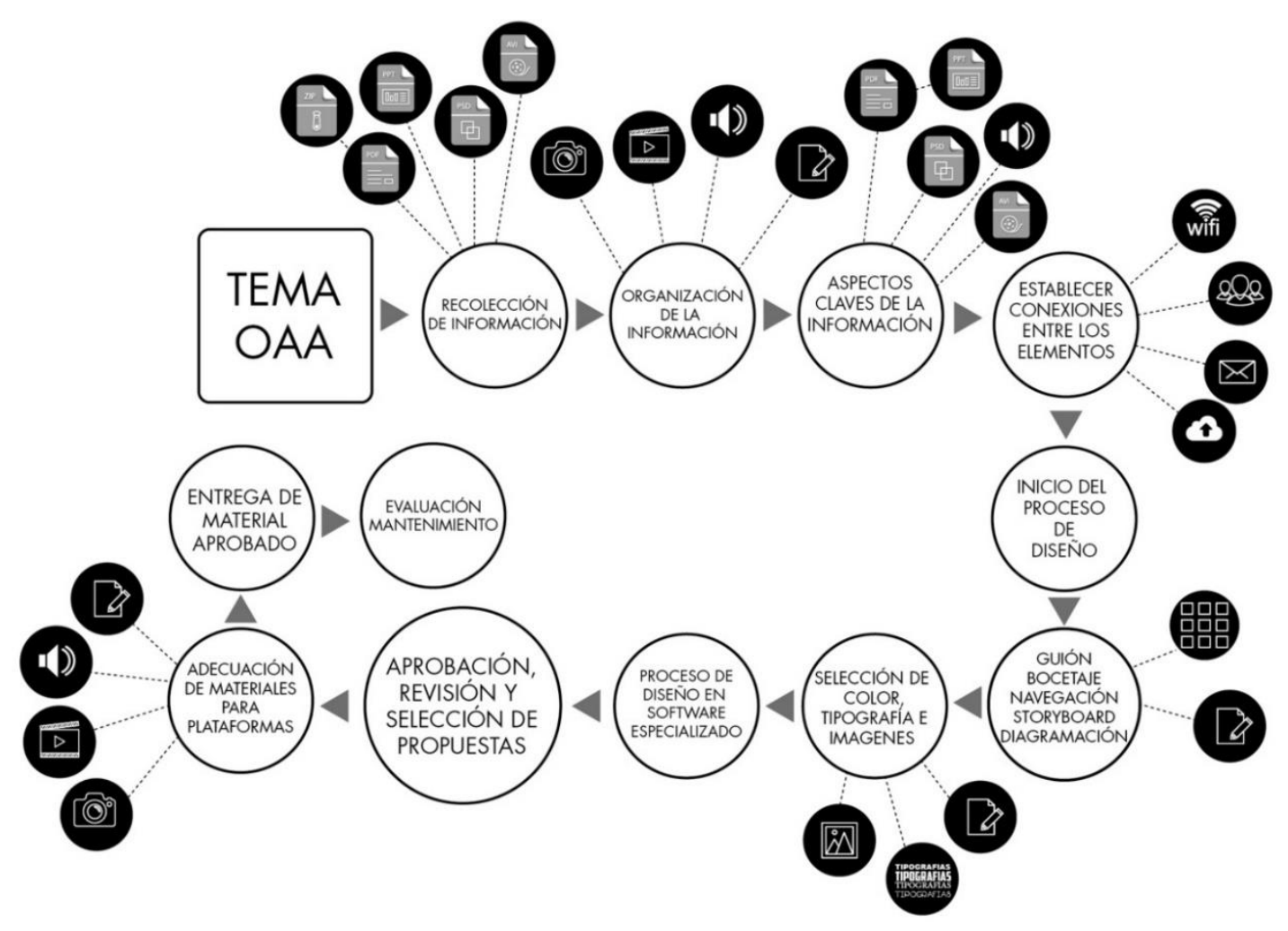

Figura 6. Propuesta del proceso de diseño gráfico para la elaboración de materiales digitales.

Más adelante, se identifican los aspectos claves de la información, el establecimiento de las conexiones entre los elementos, el inicio del proceso de diseño, guiones, bocetajes o storyboard y diagramación. Para terminar, se hace la selección de color, tipografía e imágenes, desarrollo de los elementos en software especializado, revisión y selección de propuestas basadas en ergonomía cognitiva y diseño centrado en el usuario. Asimismo, entrega de material aprobado. Por último, prepara los materiales para los repositorios de OAA o plataformas educativas. 
Se considera importante mencionar que la Figura 6 es un esquema general. La construcción de algunos materiales educativos digitales puede tener variaciones mínimas en ciertos procesos.

Además, aunque no existen reglas absolutas para el diseño de una interfaz educativa, esto no significa que deban ignorarse los principios de homogeneidad de elementos, composición y lenguaje básico del diseño de entornos virtuales educativos que favorezcan la ergonomía cognitiva.

Otra aportación del equipo de diseñadores gráficos es la elaboración de guías sencillas para que distintos participantes del proyecto para que puedan comenzar a realizar cada uno de los materiales del OAA y cumplir en tiempo y forma, como se muestra en la Figura 7.
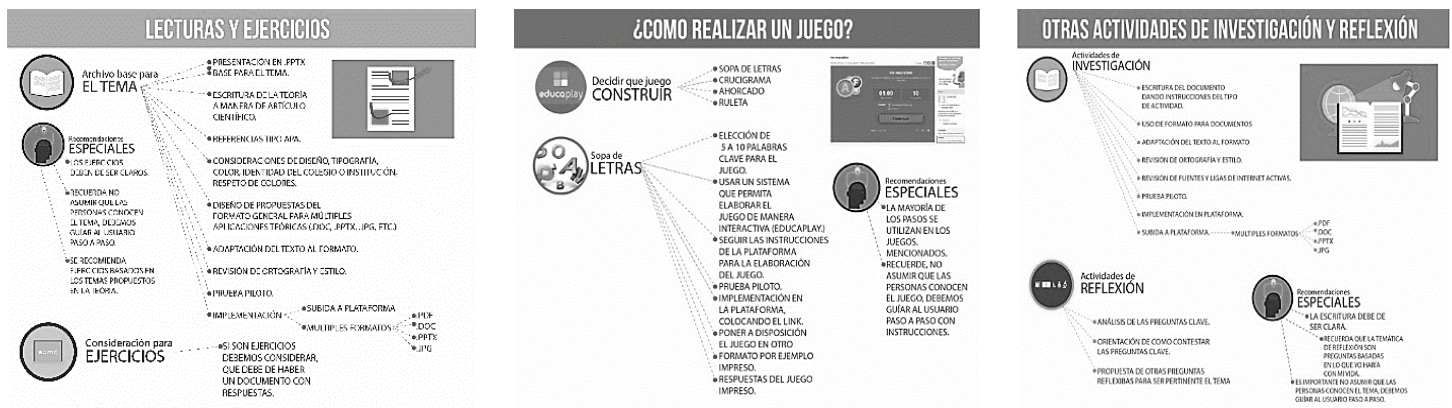

Figura 7. Guías para la elaboración de materiales digitales.

Un ejemplo del proceso para la elaboración de un video para OAA se muestra en la Figura 8.
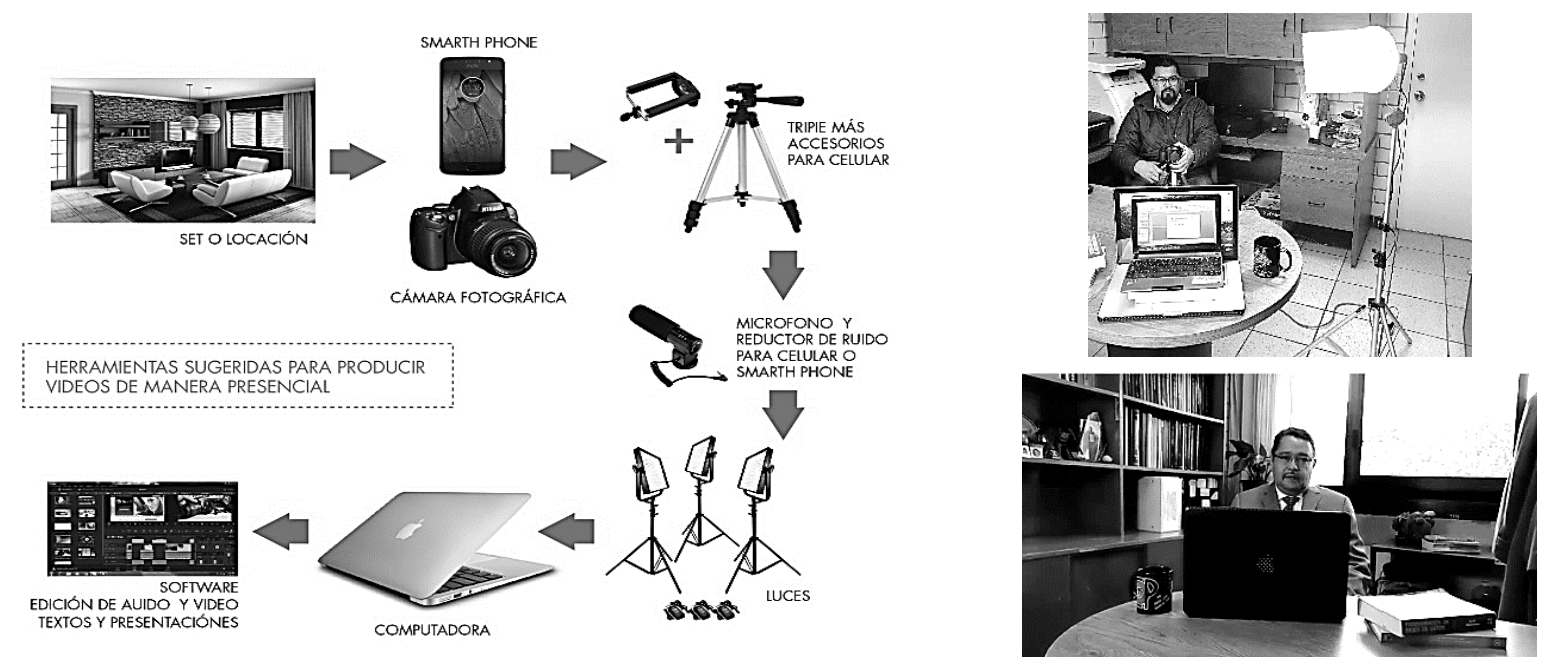

Figura 8. Elaboración de videos.

El proceso comienza con la elección del set o locación tomando en cuenta horarios de grabación con menor ruido, limpieza del sitio, adecuación de lugar para que sea lo más estético posible para la cámara. Después, la selección de equipo cámara o teléfono inteligente, trípode, micrófono y accesorios de montaje de luces. Más adelante, se integra todo el equipo haciendo sus correspondientes pruebas de foco y audio. Se graba el video considerando que los elementos incorporados estén dentro del cuadro de la cámara. 
Por último, se graba el material en el disco duro de la computadora y se edita con el software de edición de videos de licencia de código abierto, libres o con licencia de paga como imovie, movie maker, after effect, etc. Para la publicación se deben verificar las características del servidor al que se subirá, muchas veces los videos utilizan muchos bytes y se deben comprimir para ser vistos. Se puede usar HandBrake que es software libre que funciona para diferentes sistemas operativos.

Otra metodología se muestra en la Figura 9 para realizar videos utilizando software como como powtoon, after effect, premier, etc.

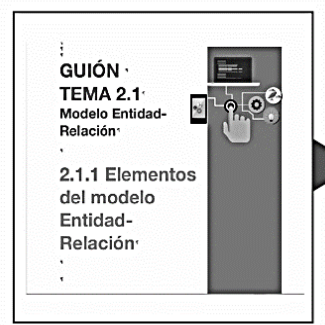

Guión

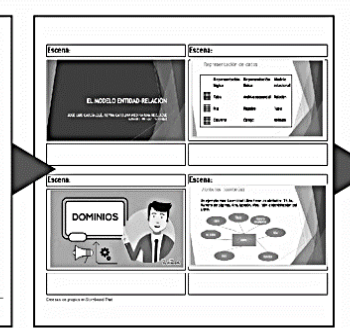

Story Board

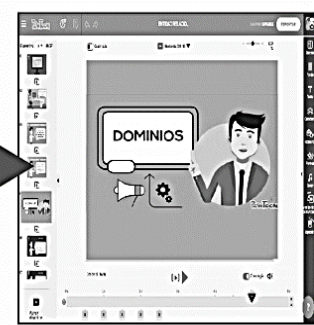

Diseño en Software

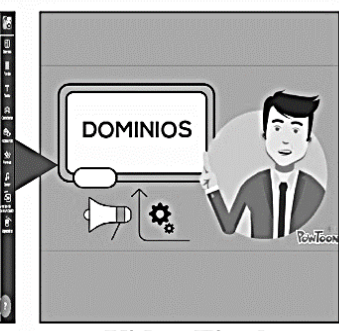

Video Final

Figura 9. Metodología para elaboración de videos con un software.

Se comienza elaborando un guión del tema que se va a tratar en el video. Después, se seleccionan los materiales para crear un story board donde se ordena se eligen personajes, animaciones, colores, tipografías, etc. Más adelante, se elabora el video utilizando el software (previamente probado) como powtoon tomando en cuenta lo propuesto en el story board. A continuación, se prueba el video para ver los tiempos de transición entre elementos y modificar errores. Para terminar, se guarda el video terminado en formatos mp4 u otros. Si se sube el video a un repositorio, se debe tomar en cuenta su tamaño, ya que muchas veces es muy grande (o pesado en bytes) y se debe reducir. Para hacerlo, puede usar un software que permita comprimir como el HandBrake.

\section{Materiales producidos para el OAA basados en los procesos de diseño gráfico}

La Figura 10 muestra ejemplos de materiales producidos basados en los procesos de diseño gráfico.

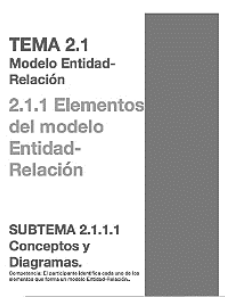

Teoría y ejemplos

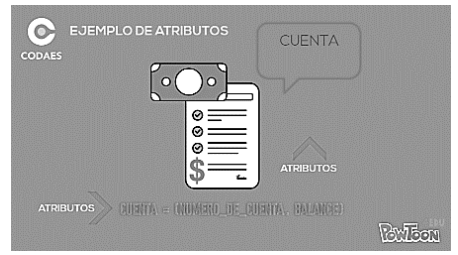

Figura 10. Materiales producidos.

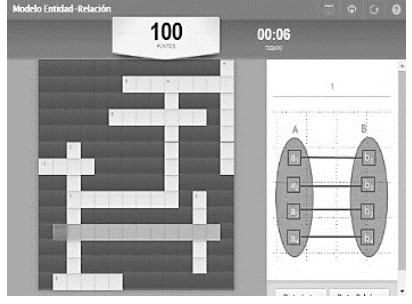

Juegos

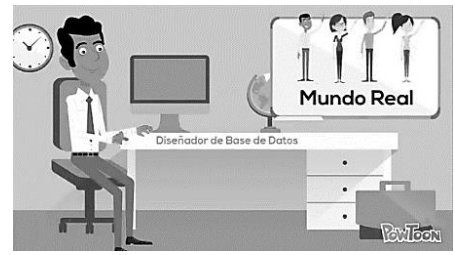

Videos

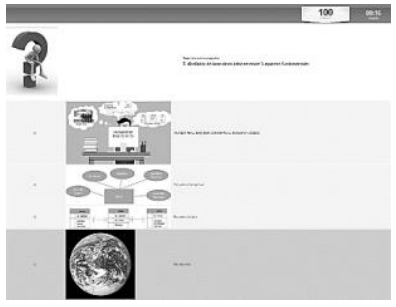

Evaluaciones

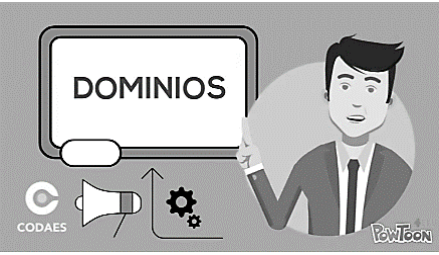


Para terminar, en la metodología PADDIEM hay constantes evaluaciones (Figuras 2 y 3), muchas de ellas realizadas con rúbricas previamente elaboradas por el equipo multidisciplinario. Dentro de PADDIEM, no se puede continuar a la siguiente etapa si no se cumple con los parámetros establecidos, esto garantiza que el OAA cumpla con el objetivo para el que fue creado. Más adelante, se tiene contemplada una investigación a discentes que ocupen los OAA y contrastarlos con otros que tomen el mismo tema de manera presencial para ver si los resultados son similares o diferentes en cuanto al proceso enseñanza-aprendizaje.

\section{Conclusiones}

Los objetivos de esta investigación se cumplieron y el supuesto no se rechaza. La metodología PADDIEM propuesta sirvió para la elaboración de objetos de de aprendizaje abiertos para ser utilizados en ciencias agrícolas. Se presentó una metodología que incluye equipos multidisciplinarios -de especialistas en el área de conocimiento, pedagogía, diseño gráfico y cómputo- donde cada experto realiza su actividad, lo que permite cumplir con los ciclos de vida de cada una de las etapas PADDIEM.

El diseño gráfico, además de aportar calidad visual y estética, apoya a la ergonomía cognitiva con el propósito de los conocimientos del OAA queden en la memoria a largo plazo. Asimismo, el diseñador gráfico cumple diversas funciones que directamente afectarán el desarrollo del diseño de los OAA. Su trabajo aporta en la adecuada percepción de los contenidos de los OAA y su permanencia en la mente de quien los utiliza. Los OAA basados en la metodología PADDIEM puede trabajar en distintos repositorios, por ejemplo, comunidades digitales para el aprendizaje en educación superior (CODAES) o Wordpress.

\section{Literatura citada}

Branson, R. K.; Wagner, B.; Rayner, G. T. 2018. Interservice procedures for instructional systems development. Task V final report. Center for Educational Technology Florida State University Tallahassee, FL 32306. 62 p.

Cacheiro, M. L. 2011. Recursos educativos TIC de información, colaboración y aprendizaje. España. Píxel-Bit Revista de Medios y Educación. 39(2):69-81 pp.

Chan, M. E.; Galeana, L.; Ramírez; M. S. 2006. Objetos de aprendizaje e innovación educativa. Trillas. México. 144 p.

Clares, J. 2011. Diseño educativo multimedia interactivo (PEMI): guía teórico-práctica. Primera edición. UNED. España. 168 p.

CODAES. 2015. Comunidades digitales para el aprendizaje en educación superior (CODAES). Objeto de aprendizaje. 1-12 pp.

Correa, L. F. 2017. Objetos de aprendizaje como estrategia. 1-4 pp.

García Aretio, L. 2013. MOOC. Objetos de aprendizaje. España. Revista Contextos Universitarios Mediados. 13(19):2-6.

García-Cué, J. L; Gutiérrez-Tapias; M.; Medina-Ramírez, M. T. B. I. R. 2017. Sistema administrador de Objetos de Aprendizaje que contienen Estilos de Aprendizaje (SIGOAEA). J. Learning Styles. 10(17):154-190. 
García- Cué, J. L.; Meraz, J. M; Medina, C.; Gutiérrez-Tapias, P. 2018. Objetos de aprendizaje abiertos fundamentados en ingeniería de Software educativo (OAAISE) para cursos de bases de datos. en diversidad educativa, armonización de competencias y transferencia al desarrollo profesional. (Ed.). Domínguez, M. C.; Medina; A.; Cacheiro; M. L. Anaya. UNED. Madrid. 124-133 pp.

González-Muñoz, E. L. 2017. Ergonomía y diseño gráfico. Elaboración de señales visuales de advertencia. México. Zincografía. Revista de comunicación y diseño. I(2):5-18. http://aprende.colombiaaprende.edu.co/sites/default/files/naspublic/manual_docentes.pdf $277 \mathrm{p}$.

Leal-Fonseca, D. E. 2008. Iniciativa colombiana de objetos de aprendizaje: situación actual y potencial para el futuro. $76-85 \mathrm{pp}$.

MENC. 2014. Ministerio de Educación Nacional de Colombia Manual de producción y gestión de contenidos educativos digitales para docentes. 13-19 pp. http://aprende.colombiaaprende. edu.co/ sites/default/files/naspublic/manual_docentes.pdf.

Meraz, J. M.; García-Cué, J. L.; Fernández, Y. M.; Jiménez, M. A.; Medina, R. C. 2018. Propuesta de un sistema de objetos de aprendizaje abiertos para cursos de bases de datos basados en ADDIE. En Diversidad Educativa, armonización de competencias y transferencia al desarrollo profesional. (Ed.). Domínguez, M. C.; Medina; A.; Cacheiro, M. L. España. Anaya-UNED. Madrid. 312-321 pp.

Montes, I. R.; García- Cué, J. L.; Del Valle, D.; Medina, C.; Escobar, J. J. 2017. Prototipo de un sistema gestor de objetos de aprendizaje para ciencias agrícolas (SIGEOACA). Rev. Mex. Cienc. Agríc. 1(8):29-38.

Moreiro, J. A.; Sánchez-Cuadrado, S.; Morato, J. 2012. Mejora de la interoperabilidad semántica para la reutilización de contenidos mediante sistemas de organización del conocimiento. Rev. Eletrôn. Bibliotec. Ciênc. Infor. 17(33):46-58.

NAVEDTRA. 2010. Naval education and training. Naval education and training command integrated learning environment course development and life-cycle maintenance. Naval Education and Training Command. EE. UU. 126 p.

Ordóñez, P.; Tennyson, R. and Lytras, M. 2015. Assessing the role of mobile technologies and distance learning in higher education. USA, Hershey PA. IGI Global. 160-162 pp.

Osóndon, Y; Castillo, P. 2006. Propuesta para el diseño de objetos de aprendizaje. Rev. Fac. Ingeniería-Universidad de Tarapacá. 14(1):36-48.

Portillo, A. D. 2017. Elaboración de objetos de aprendizaje con narrativa instruccional para un curso b-learning. Revista Razón y Palabra. 21(3)179-196.

Pratt, A. y Nunez, J. 2012. Diseño interactivo. España, Oceano. Barcelona. 223 p.

Soto, J. 2011 Repositorios semánticos de objetos de aprendizaje. USA. Raleight, Lulu. 478 p.

UNESCO. 2012. Declaración de París de 2012 sobre recursos educativos abiertos. 2 p. http://www.unesco.org/new/fileadmin/multimedia/hq/ci/ci/pdf/events/spanish_paris_oer_ declaration.pdf.

UNESCO. 2015. Guía básica de recursos educativos abiertos (REA). Elaborado por Neil Butcher para la mancomunidad del aprendizaje y la UNESCO. $150 \mathrm{p}$.

Wiley, D. 2008. The learning objects literature. In: handbook of research on educational communications and technology. (Ed.). Jonassen, D. D.; Spector, M. J.; Driscoll, M.; Merrill, M. D.; Merrienboer, J. New York: Taylor \& Francis Group. 890 p. 